\title{
Research of Gestational Risk by Primary Health Care Professionals in Brazil
}

\author{
Sebastião Junior Henrique Duarte ${ }^{1 *}$ and Ruth Andrea Dotta Miranda ${ }^{1}$ \\ Faculty of Medicine, Federal University of Mato Grosso do Sul, Brazil
}

*Corresponding author: Sebastião Junior Henrique Duarte, Fundação Oswaldo Cruz, Programa de Pós-Graduação Mestrado PROFSAÚDE. Universidade Federal de Mato Grosso do Sul, Faculdade de Medicina, Campo Grande-MS, Brazil.

To Cite This Article: Sebastião Junior Henrique Duarte, Ruth Andrea Dotta Miranda, Research of Gestational Risk by Primary Health Care Professionals in Brazil. 2020 - 11(3). AJBSR.MS.ID.001624. DOI: 10.34297/AJBSR.2020.11.001624.

Received: 眥 November 16, 2020; Published: 些 December 16, 2020

\begin{abstract}
In Brazil, the reduction of preventable causes of maternal mortality is a major challenge for health professionals. The Ministry of Health recommends the assessment of gestational risk in prenatal consultations. In this sense, a research is being carried out with the purpose of verifying how the assessment of gestational risk is made by nurses and doctors, who are the professionals who perform prenatal care. The municipality of Corumbá, Mato Grosso do Sul, was chosen to carry out the research, as it is one of the places with high indicators of maternal mortality. Data collection consists of filling in a checklist with demographic, social, environmental and health variables, all related to high gestational risk and that guide the actions to be taken to avoid complications that may lead to maternal death. This survey of how gestational risk assessment is carried out will provide subsidies to improve care for pregnant women, reduce maternal and child mortality and collaborate with the United Nations' sustainable development objectives.
\end{abstract}

\section{Introduction}

In Brazil, health care actions are determined by the Ministry of Health and are linked to international policies, such as the proposals in the sustainable development objectives of the United Nations, which have in goal 3.1 the proposal to reduce maternal mortality to 70 per cent. 100,000 live births globally by the year 2030 [1].

According to data from the Pan American Health Organization, in 2015 there were approximately 303,000 maternal deaths in Brazil and many of these deaths occurred in places with limited resources and most deaths could be prevented, mainly by qualified prenatal care, childbirth and puerperium [2].

It is noteworthy that prenatal care in Primary Health Care is performed by a multidisciplinary team, which should enhance the monitoring of maternal and fetal health conditions, thus avoiding both maternal and child morbidities and mortality. However, even with the access of pregnant women to doctors, nurses, dentists and other professionals, it was still not possible to reduce maternal mortality indicators to levels in developed countries [3].

Therefore, it is relevant studies that can carry out diagnoses capable of revealing the weaknesses in prenatal care, showing how this assistance has been carried out, as the work process must be guided by the recommendations of the Ministry of Health and the variables of classification of gestational risk are widely publicized. So, the research question is: how is the prenatal gestational risk classification performed in Primary Health Care being carried out? Which indicators are evaluated? What conducts are adopted?

\section{Research Informations}

Pregnancy is a physiological phenomenon and its evolution occurs in most cases without complications. It is a moment of change for women and, during this period, the body changes, preparing for childbirth and motherhood. Despite being a physiological phenomenon, there is a portion of pregnant women with a higher probability of unfavourable evolution, both for the fetus and for the mother, called High Risk Pregnancy.

The research starts from the assumption that, if the gestational risk is evaluated in every prenatal consultation, then the pregnant woman will have identified any situation that requires the implementation and the involvement of human, technological and economic resources that enable the resolution of the cases that can lead to maternal mortality. 
It is the responsibility of prenatal care professionals, prenatal care in accordance with scientific evidence and institutional routines aimed at the prevention, diagnosis and management of factors that can cause maternal or perinatal morbidity or mortality.

There are several factors that determine the risk during pregnancy, including some that can be detected even before a woman becomes pregnant, such as diabetes and high blood pressure. The identification of risk factors before the woman becomes pregnant allows the planning of assistance $[4,5]$.

We draw attention to the need to perform all stages of the anamnesis, general and obstetric physical examination, as well as giving the opportunity for the pregnant woman to speak if she feels something different that may require intervention. In our experience, we observed that among the common problems identified in women assisted in Primary Health Care, in addition to social and economic vulnerability, also smoking, alcoholism, use of illicit drugs such as marijuana and crack, are frequent among pregnant women and require communication skills by professionals to intervene in these problems.

There are also women with major nutritional problems, such as anemia, vitamin A deficiency and iodine. Situations that increase the risks for pregnant women and fetuses. Another important factor is violence during pregnancy, with an increased risk of miscarriage, premature birth and stillbirth.

Thus, in the global situational analysis of primary care, the intention is to reinforce measures that contribute to the early diagnosis of high-risk pregnancies and, thus, improve the rate of maternal deaths from preventable and possible causes of early interventions.
The relevance of the study is given by the potential to elaborate a diagnosis of how the classification of gestational risk occurs, based on the variables found, contributing to the reorganization of prenatal care and also to health management, with regard to the low research cost, in addition to providing a study that allows redefining work processes with a view to the quality of life of the mother and child.

This is a descriptive study and quantitative analysis. It will be performed with nurses and physicians, because they are the professionals who perform prenatal care. The research project was approved by an ethics committee in research involving human beings. Participants will sign the free and informed consent form. Data collection will be by observation and annotations in a check list containing the variables that will be marked as performed or not. The analysis will be by statistical tests and the results will compose the master's thesis of the researcher.

\section{References}

1. United Nations (2015) Sustainable Development Goals. 17 Goals to transform our world, New York, USA.

2. Panamerican Health Organization. Evidencia e Inteligencia para la Acción en Salud / Análisis de Salud, Métricas y Evidencia: Situación de Salud en las Américas: Indicadores Básicos 2018. Washington D C, Estados Unidos de América, 2018.

3. Duarte SJH, Andrade SMO, Mamede MV (2011) Maternal decision on obtaining prenatal care: a study in Brazil. Midwifery 27(2): 160-164.

4. Duarte SJH, Machado RM (2016) Essential competencies in trainning in obstetrics. Rev Panam Salud Publica 40(5): 382-387.

5. Weissgerber TL, Mudd LM (2015). Preeclampsia and diabetes. Curr Diab Rep 15(3): p9. 